\title{
Intrathecal rapamycin attenuates morphine-induced analgesic tolerance and hyperalgesia in rats with neuropathic pain
}

\author{
Ji-Tian Xu, M.D., Ph.Da, Linlin Sun, Ph.D ${ }^{\text {b }}$, Brianna Marie Lutz, B.S. ${ }^{\mathrm{c}}$, Alex Bekker, M.D, Ph.D ${ }^{\mathrm{b}}$, Yuan-Xiang Tao, M.D, \\ $\mathrm{Ph} . \mathrm{D}^{\mathrm{a}, \mathrm{b}, \mathrm{d}}$
}

${ }^{a}$ Department of Physiology \& Neurobiology, College of Basic Medicine, Zhengzhou University, Zhengzhou, Henan Province 450001, People's Republic of China ; ${ }^{b}$ Department of Anesthesiology, New Jersey Medical School, Rutgers, The State University of New Jersey, Newark, NJ 07103, USA ; 'Rutgers Graduate School of Biomedical Sciences, New Jersey Medical School, Rutgers, The State University of New Jersey, Newark, NJ 07103, USA; ${ }^{d}$ Departments of Cell Biology \& Molecular Medicine, Neurology \& Neuroscience, and Physiology \& Pharmacology, New Jersey Medical School, Rutgers, The State University of New Jersey, Newark, NJ 07103, USA

\section{Abstract}

Repeated and long-term administration of opioids is often accompanied by the initiation of opioid-induced analgesic tolerance and hyperalgesia in chronic pain patients. Our previous studies showed that repeated intrathecal morphine injection activated the mammalian target of rapamycin complex 1 (mTORC1) in spinal dorsal horn neurons and that blocking this activation prevented the initiation of morphine-induced tolerance and hyperalgesia in healthy rats. However, whether spinal mTORC1 is required for morphineinduced tolerance and hyperalgesia under neuropathic pain conditions remains elusive. We here observed the effect of intrathecal infusion of rapamycin, a specific mTORC1 inhibitor, on morphine-induced tolerance and hyperalgesia in a neuropathic pain model in rats induced by the fifth lumbar spinal nerve ligation (SNL). Continuous intrathecal infusion of morphine for one week starting on day 8 post-SNL led to morphine tolerance demonstrated by morphine-induced reduction in maximal possible analgesic effect (MPAE) to tail heat stimuli and ipsilateral paw withdrawal threshold (PWT) to mechanical stimuli in SNL rats. Such reduction was attenuated by co-infusion of rapamycin. Co-infusion of rapamycin also blocked morphine tolerance demonstrated by attenuation of morphine-induced reduction in MPAE in sham rats and morphine-induced hyperalgesia demonstrated by the reverse of morphine-induced reduction in PWT on both sides of sham rats and on the contralateral side of SNL rats. The results suggest that mTORC1 inhibitors could serve as promising medications for use as adjuvants with opioids in clinical neuropathic pain management.

Key Words: mTORC1, morphine tolerance, morphine hyperalgesia, neuropathic pain

\section{Introduction}

Neuropathic pain that results from a direct consequence of lesions or diseases is a major clinical problem. It is characterized by ongoing pain, allodynia, and hyperalgesia $(1,2)$. Current treatment of this disorder is very limited. Opioids such as morphine are the gold standard treatment for neuropathic pain (3), but repeated administrations of opioids produce severe side effects, such as analgesic tolerance and hyperalgesia. Opioid analgesic tolerance is characterized by a reduced sensitivity to the anti-nociceptive effects of opioids, requiring a higher dose to achieve the desired analgesic effect $(4,5)$, while opioidinduced hyperalgesia is a neuronal sensitization process in which opioids paradoxically produce pain hypersensitivity (4). There have been numerous investigations on the neurobiological mechanisms of opioid tolerance and hyperalgesia after chronic opioid exposure. Results mainly fall into two categories: opioid receptor signaling changes and activation of the anti-opioid nervous system. The former include phosphorylation, desensitization, internalization and downregulation of opioid receptors (6) or heterodimerization with other receptors, leading to an enhanced perception of pain (7). The latter includes enhanced excitatory primary afferents, descending spinal facilitation, glial cell activation, and up-regulation of dorsal horn intracellular nNOS, PKC $\gamma$, and CaMK II signaling (8). All of these changes may merge and act through a translation modulation process, contributing to the mechanism of opioid-induced tolerance and hyperalgesia.

The mammalian target of rapamycin (mTOR) is a serinethreonine protein kinase, which forms 2 distinct protein complexes, mTOR complex 1 (mTORC1) and mTORC2. 
In the mammalian nervous system, mTOR1 is composed of raptor, mLST8, and mTOR and is generally believed to control the translation of most proteins by phosphorylation of specific downstream effectors $(9,10)$. Our previous work has demonstrated that repeated intrathecal morphine injections activated mTORC1 in rat spinal dorsal horn neurons $(9,11)$. This activation was triggered through cellular membrane $\mu$ opioid receptor and mediated by intracellular signals PI3K/ Akt. Moreover, intrathecal administration of a specific mTOR inhibitor attenuated both the induction and maintenance of morphine tolerance and hyperalgesia $(9,11)$. These effects could be interpreted by the attenuation of morphine-induced increases in translation initiation activity, nascent protein synthesis, and the expression of some known key toleranceassociated proteins, such as neuronal NOS (nNOS), in the dorsal horn $(9,11)$. Our findings indicate that spinal mTORC1 plays a key role in the initiation and maintenance of morphine-induced tolerance and hyperalgesia. However, all of these experiments were carried out on healthy animals. Given that opioids are used in patients with chronic pain, the goal of the present study was to further examine if spinal mTORC1 plays a key role in the initiation of morphineinduced tolerance and hyperalgesia under neuropathic pain conditions.

\section{Materials and methods}

\section{Animals}

All animal experimental procedures follow ethical guidelines produced by the National Institutes of Health and the International Association for the Study of Pain. Animal protocols are approved by the Rutgers New Jersey Medical School Animal Care and Use Committee. Male SpragueDawley Rats weighing 180-250g were housed separately with food and water provided ad libitum on a $12 \mathrm{~h}$ light/ $12 \mathrm{~h}$ dark cycle. Animals were exposed to habituation for 2 days prior to use. Behavioral tests were completed in a blinded manner. Every effort was made to minimize the amount of animals used and their suffering.

Surgical procedures and drug infusion

Rats underwent a modified unilateral L5 spinal nerve ligation (SNL) as previously described $(12,13,14)$. In brief: after the animals were anesthetized by isoflurane, the left L6 transverse process was removed to expose the L4 and L5 spinal nerves. After isolation of the left L5 spinal nerve, a tight ligature was made with 3-0 silk and the nerve was transected distal to this ligature. In sham-operated rats, the left L5 spinal nerve was isolated, but remained intact with no ligature or transection.

Rats underwent intrathecal catheter implantation for drug delivery in the same manner as described previously (15). In brief: while under isoflurane-induced anesthesia, a laminectomy of the L5 vertebra was performed and the dura was cut. At the level of the L4/5 spinal cord, a polyethylene-10 catheter was inserted into the subarachnoid

Transl Perioper \& Pain Med 2015; 2 (2) space. Following catheter implantation, animals underwent 7 days of recovery prior to SNL or sham surgery. A miniosmotic pump (Alzet, Cupertino, CA, USA) connected to the intrathecal catheter was used for continuous infusion of drug at a rate of $1 \mu \mathrm{l} / \mathrm{h}$. Rats were divided into five groups for drug delivery: saline plus vehicle (10\% DMSO in saline), saline plus rapamycin (a selective inhibitor of mTORC1, $1 \mu \mathrm{g} / \mathrm{h}$, Sigma), morphine (15 $\mu \mathrm{g} / \mathrm{h}$, West-Ward, Eatontown, $\mathrm{NJ})$ plus rapamycin, morphine plus ascomycin $(1 \mu \mathrm{g} / \mathrm{h}$, Sigma), or morphine plus vehicle. Beginning 8 days after SNL or Sham surgery, continuous intrathecal infusion was conducted for 24 hours a day for 7 days from day 8 to day 14. Given that ascomycin (an analogue of rapamycin) does not inhibit mTORC1 activity $(16,17)$, we used ascomycin to confirm the specificity of the action of rapamycin on mTORC1. Rats with neurological deficits were excluded from the study. The location of the intrathecal catheter was confirmed after completion of the experiments.

\section{Behavioral testing}

Tail flick testing was carried out as described previously (9). In brief, tail flick latency to noxious heat was measured on day 7 (the day before continuous intrathecal morphine injection), and days $8,10,12$, and 14 (during continuous morphine injection) using an Analgesic Meter (Model 33B Tail Flick Analgesia Meter, IITC Life Science, Woodland Hills, CA, USA). The cut-off time was $10 \mathrm{~s}$. Three trials were conducted for each rat with an interval of $1 \mathrm{~min}$. Morphine's maximal possible analgesic effect (MPAE) was determined using the following equation: MPAE $=[($ posttreatment pretreatment $) /(10$ - pretreatment $)] \times 100 \%$.

Mechanical paw withdrawal thresholds were determined using the up-down method following a previously described procedure $(13,14)$. Mechanical paw withdrawal thresholds (PWTs) were measured 1 day before SNL, and on days 7, $8,10,12$, and 14 during continuous morphine injection. In brief, each rat was placed in an individual Plexiglas chamber on an elevated mesh screen. Von Frey hairs in log increments of force $(0.407,0.692,1.202,2.041,3.63,5.495$, $8.511,15.14,26.0 \mathrm{~g})$ were applied to the plantar surface of the left and right hind paws, beginning with the $2.041 \mathrm{~g}$ Von Frey hair. If the rat exhibited a positive response, the next smaller von Frey hair was used; if a negative response was observed, the next larger von Frey hair was used. The test was terminated under one of two conditions: (i) a negative response was obtained with the highest force (26.0-g) or (ii) three stimuli were applied after the first positive response. The formula provided by Dixon (18-20) was used to convert the patterns of positive and negative responses to a $50 \%$ threshold value.

Following the behavioral tests described above, locomotor tests were conducted according to previously described methods $(13,14)$ These tests included: (1) Placing reflex: while holding the rat with its hind limbs slightly 
lower than its forelimbs, the experimenter recorded whether

Fig. 1
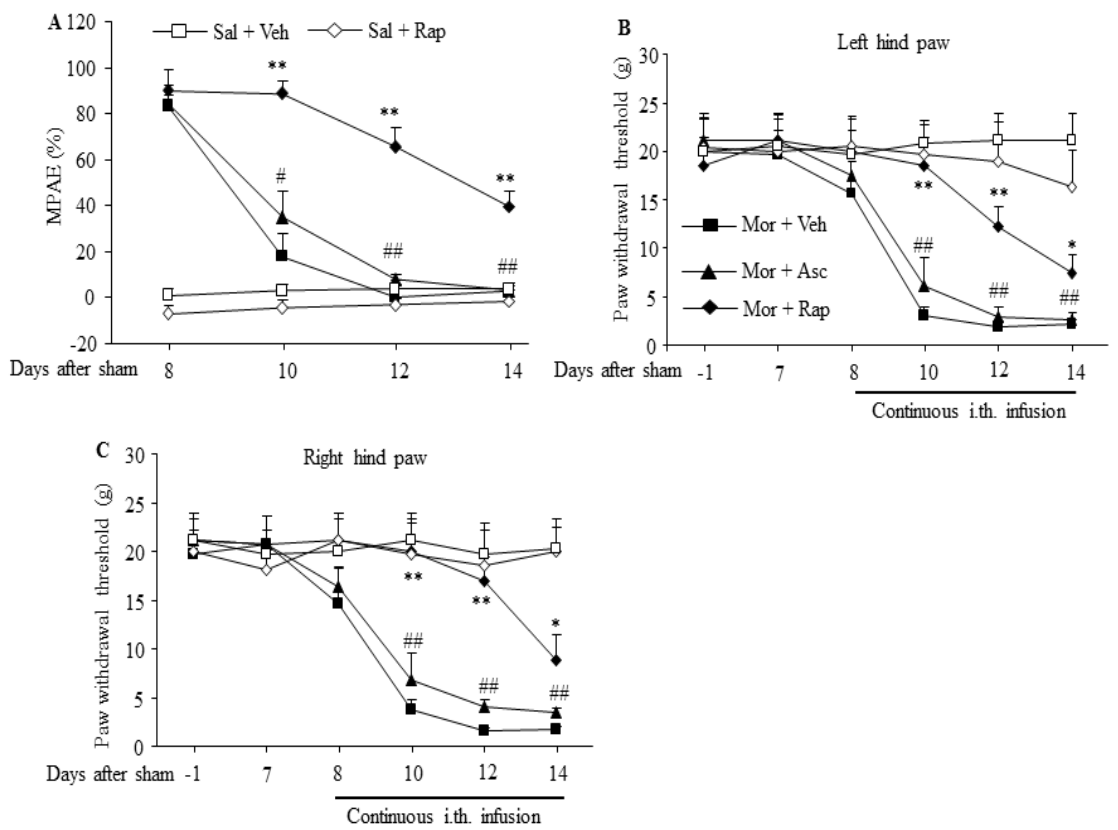

the rat placed its hind paws on the table surface reflexively when the hind paw dorsal surfaces made contact with the edge of the table; (2) Grasping reflex: the rat was placed on a wire grid, and the experimenter recorded whether the hind paws grasped the wire on contact; (3) Righting reflex: The experimenter placed the rat on its back on a flat surface, and recorded whether the rat immediately assumed an upright position. Five trials were conducted for each test and scores for placing, grasping, and righting reflexes were based on counts of each normal reflex for each trial. In addition, the rats' general behaviors, including spontaneous painassociated activity, were observed.

\section{Statistical analysis}

All results are given as mean \pm SEM. Data were analyzed using one-way ANOVA tests followed by Dunnett post-tests (comparison of all other columns vs. control column). Statistical tests were conducted using GraphPad Prism 5.0 software. P values of less than 0.05 were considered statistically significant.

\section{Results}

Effect of intrathecal infusion of rapamycin on the development of morphine-induced analgesic tolerance and hyperalgesia in sham-operated rats

In sham-operated rats, morphine tolerance was induced by continuous intrathecal infusion of morphine at a rate of $15 \mu \mathrm{g} / \mu \mathrm{l} / \mathrm{h}$ for 7 days from day 8 to 14 . The MPAEs of the morphine plus vehicle group were significantly and time-dependently decreased on day 10,12 and 14 during continuous morphine infusion $\left(\mathrm{F}_{3,16}=39.3\right.$, \# $p<0.05$, \#\#
Figure 1. Intrathecal co-infusion of rapamycin prevented the development of morphine-induced analgesic tolerance and hyperalgesia in sham-operated rats. $n=5-7$ rats per group. Morphine (Mor) was infused intrathecally at a rate of $15 \mu \mathrm{g} / \mu \mathrm{l} / \mathrm{h}$ for 7 days from day 8 to 14 after sham surgery. Intrathecal co-infusion of rapamycin (Rap; $1 \mu \mathrm{g} / \mu \mathrm{l} / \mathrm{h}$ ), but not ascomycin (Asc; $1 \mu \mathrm{g} / \mu \mathrm{l} / \mathrm{h})$, attenuated morphine-induced reductions in morphine's maximal possible analgesic effects (MPAEs) (A) and in paw withdrawal thresholds on left (B) and right (C) hind paws on day 10,12 and 14. Sal: saline. Veh: vehicle (10\% DMSO in saline). \# $\mathrm{p}<0.05$, \#\# $\mathrm{p}<0.01$ vs. the corresponding baseline ${ }^{*} \mathrm{p}<0.05,{ }^{* *} \mathrm{p}<0.01$ vs. the corresponding time points in the morphine plus vehicle group (one-way ANOVA, followed by Dunnett post-test). $p<0.01 v s$. the corresponding baseline; Fig. 1A). However, when these sham-operated rats received morphine plus rapamycin, the decrease of MPAE was effectively reversed. Compared with the morphine plus vehicle group, the MPAEs of the morphine plus rapamycin group were significantly increased by 4.9-fold, 14.7-fold, and 12.9 fold on day 10, 12, and 14 after morphine infusion, respectively, $\left(\mathrm{F}_{4,20}=25.86\right.$ for day $10, \mathrm{~F}_{4,20}=32.84$ for day $12, \mathrm{~F}_{4,20}=14.5$ for day 14 . ** $p<0.01 v s$. the corresponding time points in the morphine plus vehicle group; Fig. 1A). In contrast, the sham-operated rats that received continuous infusion of morphine plus ascomycin still displayed a remarkable decrease in MPAEs. No statistical difference was found between the morphine plus vehicle group and the morphine plus ascomycin group (Fig. 1A). The continuous infusion of rapamycin alone in the saline plus rapamycin group did not alter the basal response to heat stimulation (Fig. 1A).

Continuous intrathecal infusion of morphine also led to hyperalgesia demonstrated by significant and timedependent reductions in PWTs in response to mechanical stimuli in the morphine plus vehicle group on day 10,12 and 14 during continuous morphine infusion on bilateral hind paws of sham-operated rats $\left(\mathrm{F}_{5,24}=21.69\right.$ for ipsilateral side, and $\mathrm{F}_{5,24}=16.17$ for contralateral side. \#\# $p<0.01 \mathrm{vs}$. the corresponding baseline; Fig.1B, C). These reductions were significantly attenuated by co-administration of rapamycin in the morphine plus rapamycin group $\left(\mathrm{F}_{4,20}=10.37\right.$ for day $10, \mathrm{~F}_{4,20}=13.63$ for day $12, \mathrm{~F}_{4,20}=14.39$ for day 14 . $* p<0.05, * * p<0.01 v s$. the corresponding time points 

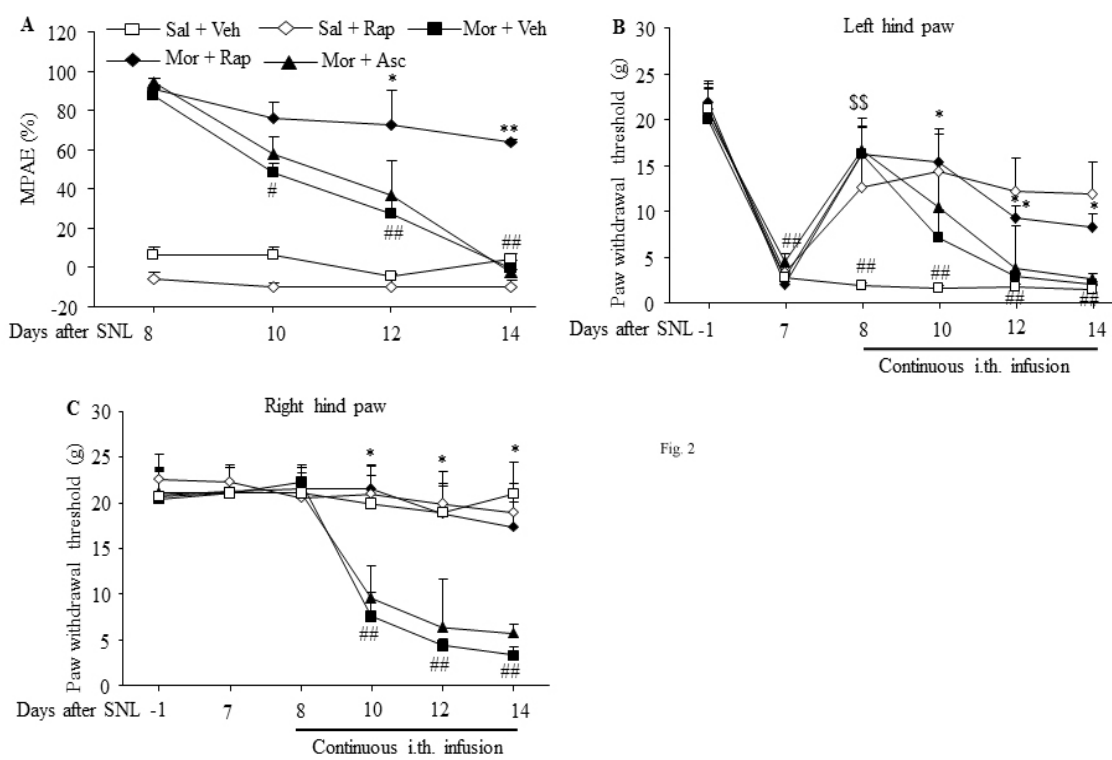

Fig 2

Figure 2. Intrathecal co-infusion of rapamycin prevented the development of morphine-induced analgesic tolerance and hyperalgesia in SNL rats. $\mathrm{n}=5-6$ rats per group. Morphine (Mor) was infused intrathecally at a rate of $15 \mu \mathrm{g} / \mu \mathrm{l} / \mathrm{h} \mathrm{for} 7$ days from day 8 to 14 after SNL surgery. Intrathecal co-infusion of rapamycin (Rap; $1 \mu \mathrm{g} / \mu \mathrm{l} / \mathrm{h}$ ), but not ascomycin (Asc; 1 $\mu \mathrm{g} / \mu \mathrm{l} / \mathrm{h}$ ), attenuated the morphine-induced reductions in maximal possible analgesic effects (MPAEs) (A) and paw withdrawal thresholds on the ipsilateral (B) and contralateral (C) sides on day 10, 12 and 14. Intrathecal rapamycin alone in the rapamycin plus saline group also attenuated the SNL-induced decrease in the paw withdrawal threshold in response mechanical stimulation on the ipsilateral side on day 8-14 post-surgery in SNL rats (B). Sal: saline. Veh: vehicle (10\% DMSO in saline). $\# \mathrm{p}<0.05, \# \# \mathrm{p}<0.01$ vs. the corresponding baseline. ${ }^{\star} \mathrm{p}<0.05,{ }^{\star \star} \mathrm{p}<0.01$ vs. the corresponding time points in the morphine plus vehicle group. $\$ \$ p<0.01$ vs. the corresponding time points in the saline plus vehicle group (one-way ANOVA, followed by Dunnett post-test).

in the morphine plus vehicle group; Fig.1B, C), not by coadministration of ascomycin in the morphine plus ascomycin group (Fig. 1B, C). As expected, the continuous infusion of rapamycin alone in the saline plus rapamycin group did not alter the basal response to mechanical stimulation on either hindpaw in sham-operated rats (Fig. 1B, C).

Effect of intrathecal infusion of rapamycin on the development of morphine analgesic tolerance and hyperalgesia in L5 SNL rats

Continuous intrathecal infusion of morphine for 7 days starting on day 8 after SNL surgery produced morphine analgesic tolerance. The MPAEs in the morphine plus vehicle group were significantly decreased by $42.6 \%$ (\# $p<0.05$ ), 68.8\% (\#\# $p<0.01$ ), and 87.4\% (\#\# $p<0.01)\left(\mathrm{F}_{3,16}=38.789\right)$ on days 10,12 , and 14 during continuous morphine infusion, respectively, as compared to day 8 , the first day of morphine infusion (Fig. 2A). The reductions on days 12 and 14 were effectively prevented by co-administration of rapamycin in the morphine plus rapamycin group $\left(\mathrm{F}_{4,20}=35.34\right.$ for day 10 , $\mathrm{F}_{4,20}=10.47$ for day $12, \mathrm{~F}_{4,20}=39.34$ for day $14 . * p<0.05, * *$ $p<001 v s$. the corresponding MPAEs in the morphine plus vehicle group), although no significant difference was found on day 10 after morphine infusion (Fig. 2A). As expected, coinfusion of ascomycin in the morphine plus ascomycin group did not affect morphine-induced reductions in the MPAEs on day 10, 12, and 14 after morphine infusion in SNL rats (Fig. 2A). The continuous co-infusion with rapamycin alone in the saline plus rapamycin group did not change the MPAEs during the observation period in SNL rats (Fig. 2A).

In our rat SNL model of neuropathic pain, development of mechanical allodynia on the ipsilateral side is made evident by significant decreases in PWTs on day 7 to 14 after SNL in the saline plus vehicle group $\left(\mathrm{F}_{5,24}=10.58\right.$. \#\# $p<0.01 \mathrm{vs}$. the corresponding baseline; Fig. 2B). Intrathecal infusion of morphine for 1 day on day 8 in the morphine plus vehicle group produced a significant analgesic effect on SNLinduced mechanical allodynia on the ipsilateral side. The PWT in the morphine plus vehicle group was increased by 8.9-fold as compared to that in the saline plus vehicle group $\left(\mathrm{F}_{4,20}=4.745\right.$ for day 8. $\$ \$ p<0.01$; Fig. 2B). However, the PWT in the morphine plus vehicle group was markedly reduced during continuous morphine infusion (Fig. 2B). This rapid decrease in morphine analgesic effect indicates successful induction of morphine analgesic tolerance in SNL rats. These reductions were significantly blocked on day 10 , 12 , and 14 by co-infusion of rapamycin in the morphine plus rapamycin group, not by co-infusion of ascomycin in the morphine plus ascomycin group, in $\mathrm{SNL}$ rats $\left(\mathrm{F}_{4,20}\right.$ 
$=3.247$ for day $10, \mathrm{~F}_{4,20}=2.832$ for day $12, \mathrm{~F}_{4,20}=7.105$ for day 14. ${ }^{*} p<0.05$ or $* * p<0.01$ vs. the corresponding time points in the morphine plus vehicle group; Fig. 2B). Additionally, continuous intrathecal infusion of rapamycin alone starting on day 8 in the rapamycin plus vehicle group clearly ameliorated SNL-induced mechanical allodynia demonstrated by the reverse of SNL-induced decreases in PWTs on the ipsilateral side from day 8 to 14 after SNL (Fig. 2B).

Consistent with previous observations $(13,14)$, SNL did not alter basal contralateral PWTs during the observation period in the saline plus vehicle group (Fig. 2C). Similar to the sham-operated rats (Fig. 1A and B), continuous intrathecal infusion of morphine starting on day 8 in the morphine plus vehicle group produced significant reductions in PWT in response to mechanical stimuli on day 10,12 and 14 after morphine infusion on the contralateral hind paw of SNL rats $\left(\mathrm{F}_{5,24}=17.33\right.$. \#\# $p<0.01$ vs. the corresponding baseline; Fig. $2 \mathrm{C})$, an indication of morphine-induced hyperalgesia. These reductions were abolished by co-infusion of rapamycin in the rapamycin plus morphine group $\left(\mathrm{F}_{4,20}=3.31\right.$ for day $10, \mathrm{~F}_{4,20}$ $=2.918$ for day $12, \mathrm{~F}_{4,20}=7.724$ for day $14 . * * p<0.01 v s$. the corresponding time points in the morphine plus vehicle group; Fig. 2C), but not of ascomycin in the ascomycin plus morphine group (Fig. 2C) on the contralateral side of SNL rats. As expected, continuous intrathecal infusion of rapamycin alone in the rapamycin plus saline group did not alter basal PWTs during the observation period (Fig. 2C).

Effect of intrathecal infusion of rapamycin on locomotor functions

To exclude the possibility that the effect of intrathecal infusion of rapamycin on behavioral responses described above was caused by impaired locomotor functions (or reflexes), we finally examined locomotor functions of experimental rats. As shown in Table 1, the rats injected with saline plus vehicle, saline plus rapamycin, morphine plus vehicle, morphine plus ascomycin, or morphine plus rapamycin displayed normal locomotor functions, including placing, grasping, and righting reflexes. Convulsions and hypermobility were not observed in any of the injected rats.

\section{Discussion}

In the present study, we demonstrated that spinal mTORC1 inhibition by intrathecal rapamycin delayed morphine analgesic tolerance, attenuated morphine-induced hyperalgesia, and blocked SNL-induced mechanical allodynia in neuropathic pain rats. We have previously shown that dorsal horn mTORC1 activation contributes to the development and maintenance of chronic morphine tolerance and hyperalgesia via mTORC1-mediated increase in dorsal horn protein translation in healthy rats. Here, our further observations suggest that spinal mTORC1 is likely a novel target for therapeutic prevention of opioid tolerance and hyperalgesia in neuropathic pain management.

The mechanisms of morphine tolerance and hyperalgesia are generally considered to arise from adaptive changes within the peripheral and central nervous systems (11). The initiation of morphine tolerance and hyperalgesia may involve adaptive modifications of the $\mu$-opioid receptor, particularly desensitization and downregulation, as well as adaptive changes in neuronal circuits, including activation of the anti-opioid system $(6,11,21,22)$. These adaptive changes may converge on the protein translation process $(11,23)$. Repeated intrathecal injections of morphine were reported to increase translational activity and new protein synthesis in the spinal dorsal horn, which could be blocked by spinal cord mTORC1 inhibition $(9,11)$. We show here that intrathecal co-infusion of rapamycin blocked morphineinduced analgesic tolerance and hyperalgesia in the shamoperated or SNL rats. These findings support previous observations in healthy rats in that repeated intrathecal morphine elevated spinal mTORC1 activity and inhibiting this activity attenuated both the induction and maintenance of morphine tolerance and hyperalgesia (9-11). It is likely that spinal mTORC1 participates in mechanisms that underlie the development and maintenance of opioid tolerance and hyperalgesia under neuropathic pain conditions.

Interestingly, the time course in the development of morphine-induced tolerance and hyperalgesia in SNL

Table 1: Mean (SEM) changes in locomotor function

\begin{tabular}{lccc}
\hline \multicolumn{1}{c}{ Group } & Placing & $\begin{array}{c}\text { Functional test } \\
\text { Grasping }\end{array}$ & Righting \\
\hline Saline + vehicle & $5(0)$ & $5(0)$ & $5(0)$ \\
Saline + rapamycin & $5(0)$ & $5(0)$ & $5(0)$ \\
Morphine + vehicle & $5(0)$ & $5(0)$ & $5(0)$ \\
Morphine + ascomycin & $5(0)$ & $5(0)$ & $5(0)$ \\
Morphine + rapamycin & $5(0)$ & $5(0)$ & $5(0)$ \\
\hline
\end{tabular}

$\mathrm{n}=5$ /group. 5 trials. 
rats is similar to that in sham rats, indicating that SNL does not alter the initiation of analgesic tolerance to morphine and the development of morphine-induced hyperalgesia. It is very likely that the role of mTORC1 in the mechanisms of SNL-induced neuropathic pain development is different from the underlying mechanisms of morphine tolerance and hyperalgesia. Indeed, chronic morphineinduced dorsal horn mTOR activation occurs through the $\mu$-opioid receptor-triggered PI3K/ AKT pathway. The activated $\mathrm{mTORC} 1$ phosphorylates its downstream targets; eukaryotic initiation factor 4E-binding proteins (4E-BPs) and $\mathrm{p} 70$ ribosomal $\mathrm{S} 6$ protein kinases (S6Ks), and gates the synthesis of many individual proteins that participate in the development and maintenance of morphine tolerance and hyperalgesia $(9,11)$. In contrast, SNL did not alter the basal level of spinal phosphorylated mTORC1 (15), although behavioral observations from the present study and those of others showed that local, intrathecal, or systemic administration of rapamycin alleviated neuropathic pain (24-27). The mechanisms of how mTORC1 is involved in neuropathic pain are elusive, but the anti-nociceptive effect of rapamycin may be related to its interaction with pro-nociceptive sodium channels, resulting in functional reduction of these channels, or it likely acts as an effective immunosuppressive agent activating autophagy, decreasing cytokine release and suppressing immune-cell-derived microglial cells $(28,29)$. It appears that the mechanisms by which mTORC1 participates in neuropathic pain and morphine-induced tolerance/hyperalgesia are distinct. However, these mechanistic differences do not affect the use of rapamycin and its analogues as adjuvants with opioids in neuropathic pain management.

Although a previous study indicated that microgliamediated disruption of neuronal $\mathrm{Cl}^{-}$homeostasis was necessary for the development of morphine hyperalgesia, but not of morphine tolerance (30), it is generally considered that common cellular mechanisms are shared under the conditions of morphine-induced tolerance and hyperalgesia because both can be blocked by the same manipulation $(9,11,31,32)$. The present study indicated that intrathecal co-infusion of rapamycin attenuated both morphineinduced tolerance and hyperalgesia in neuropathic pain, which further supports the notion that targeting shared mechanisms provide not only a strategy to enhance the utility of opioids to overcome opioid tolerance but also serve as an avenue to reduce other opioid-induced side effects, such as constipation, respiratory inhibition, and over sedation.

In conclusion, our findings strongly support the role of spinal mTORC1 in morphine- induced analgesic tolerance and hyperalgesia in neuropathic pain. mTORC1 inhibitors are FDA-approved clinical drugs for organ transplantation and cancer treatment. Therefore, mTORC1 inhibitors could be promising drugs for use as adjuvants with opioids in treating chronic pain including neuropathic pain and cancer pain.

\section{Conflict Interests Disclosure:}

The authors have no conflicting interests to disclose.

\section{Corresponding Author:}

Yuan-Xiang Tao, MD, PhD

Department of Anesthesiology

Rutgers New Jersey Medical School, Rutgers, The State University of New Jersey

185 S. Orange Ave., MSB, F-548, Newark, NJ 07103.

Tel: +1-973-972-9812; Fax: +1-973-972-1644

E-mail: yuanxiang.tao@njms.rutgers.edu

\section{Editor}

Renyu Liu, MD; PhD; Associate Professor

Director of Preoperative Medicine; Department of Anes-

thesiology and Critical Care; Perelman School of Medicine at the University of Pennsylvania.

336 John Morgan building, 3620 Hamilton Walk, Philadelphia, PA 19104

Email: 1iur@uphs.upenn.edu

\section{Disclosure of Funding}

This work was supported by grants from the National Institutes of Health, Bethesda, Maryland (NS072206, HL117684, and DA033390) and the Rita Allen Foundation, Princeton, New Jersey.

\section{Additional publication details}

Journal short name: Transl Perioper \& Pain Med

Received Date:Aug 13, 2015

Accepted Date: Aug 23, 2015

Published Date: Aug 252015

Transl Perioper \& Pain Med 2015; 2(2):27-34

\section{Citation and Copyright}

Citation: Xu JT, Sun L, Lutz BM, Bekker A, Tao YX. Intrathecal rapamycin attenuates morphine-induced analgesic tolerance and hyperalgesia in rats with neuropathic pain. Transl Perioper \& Pain Med 2015; 2(2): 27-34

Copyright: (C) $2015 \mathrm{Xu}$ JT et al. This is an open-access article distributed under the terms of the Creative Commons Attribution 
License, which permits unrestricted use, distribution, and reproduction in any medium, provided the original author and source are credited.

\section{References}

1. Campbell JN, Meyer RA. Mechanisms of neuropathic pain. Neuron 2006;52:77-92. PMID: 17015228

2. Woolf CJ. Central sensitization: implications for the diagnosis and treatment of pain. Pain 2011;152:S2-15. PMID: 20961685

3. Yu G, Zhang FQ, Tang SE, Lai MJ, Su RB, Gong ZH. Continuous infusion versus intermittent bolus dosing of morphine: a comparison of analgesia, tolerance, and subsequent voluntary morphine intake. J Psychiatr Res 2014;59:161-6. PMID: 25193460

4. Bekhit MH. Opioid-induced hyperalgesia and tolerance. Am J Ther 2010;17:498-510. PMID: 20844348

5. Rozenfeld R, Abul-Husn NS, Gomez I, Devi LA. An emerging role for the delta opioid receptor in the regulation of mu opioid receptor function. Sci World J 2007;7:64-73. PMID: 17982578

6. Ueda $\mathrm{H}$, Ueda M. Mechanisms underlying morphine analgesic tolerance and dependence. Front Biosci (Landmark Ed) 2009;14:5260-72. PMID: 19482614

7. Szabo I, Chen XH, Xin L, Adler MW, Howard OM, Oppenheim JJ, Rogers TJ. Heterologous desensitization of opioid receptors by chemokines inhibits chemotaxis and enhances the perception of pain. Proc Natl Acad Sci U S A 2002;99:10276-81. PMID: 12130663

8. Mayer DJ, Mao J, Holt J, Price DD. Cellular mechanisms of neuropathic pain, morphine tolerance, and their interactions. Proc Natl Acad Sci U S A 1999;96:7731-6. PMID: 10393889

9. Xu JT, Zhao JY, Zhao X, Ligons D, Tiwari V, Atianjoh FE, Lee CY, Liang L, Zang W, Njoku D, Raja SN, Yaster M, Tao YX. Opioid receptortriggered spinal mTORC1 activation contributes to morphine tolerance and hyperalgesia. J Clin Invest 2014;124:592-603. PMID: 24382350

10. Xu JT, Zhao X, Yaster M, Tao YX. Expression and distribution of mTOR, p70S6K, 4E-BP1, and their phosphorylated counterparts in rat dorsal root ganglion and spinal cord dorsal horn. Brain Res 2010;1336:46-57. PMID: 20399760

11. Lutz BM, Nia S, Xiong M, Tao YX, Bekker A. mTOR, a new potential target for chronic pain and opioid-induced tolerance and hyperalgesia. Mol Pain 2015;11:32. PMID: 26024835

12. Kim KJ, Yoon YW, Chung JM. Comparison of three rodent neuropathic pain models. Exp Brain Res 1997;113:200-6. PMID: 9063706

13. Fan L, Guan X, Wang W, Zhao JY, Zhang H,
Tiwari V, Hoffman PN, Li M, Tao YX. Impaired neuropathic pain and preserved acute pain in rats overexpressing voltage-gated potassium channel subunit Kv1.2 in primary afferent neurons. Mol Pain 2014;10:8. PMID: 24472174

14. Zhao X, Tang Z, Zhang H, Atianjoh FE, Zhao JY, Liang L, Wang W, Guan X, Kao SC, Tiwari V, Gao YJ, Hoffman PN, Cui H, Li M, Dong X, Tao YX. A long noncoding RNA contributes to neuropathic pain by silencing Kcna2 in primary afferent neurons. Nat Neurosci 2013;16:1024-31. PMID: 23792947

15. Liang L, Tao B, Fan L, Yaster M, Zhang Y, Tao YX. mTOR and its downstream pathway are activated in the dorsal root ganglion and spinal cord after peripheral inflammation, but not after nerve injury. Brain Res 2013;1513:17-25. PMID: 23583278

16. Lisi L, Aceto P, Navarra P, Dello Russo C. mTOR kinase: a possible pharmacological target in the management of chronic pain. Biomed Res Int 2015;2015:394257. PMID: 25685786

17. Yip CK, Murata K, Walz T, Sabatini DM, Kang SA. Structure of the human mTOR complex I and its implications for rapamycin inhibition. Mol Cell 2010;38:768-74. PMID: 20542007

18. Chaplan SR, Bach FW, Pogrel JW, Chung JM, Yaksh TL. Quantitative assessment of tactile allodynia in the rat paw. J Neurosci Methods 1994;53:55-63. PMID: 7990513

19. Dixon WJ. Efficient analysis of experimental observations. Annu Rev Pharmacol Toxicol 1980;20:441-62. PMID: 7387124

20. Dixon WJ. Staircase bioassay: the up-and-down method. Neurosci Biobehav Rev 1991;15:47-50. PMID: 2052197

21. Ossipov MH, Lai J, King T, Vanderah TW, Porreca F. Underlying mechanisms of pronociceptive consequences of prolonged morphine exposure. Biopolymers 2005;80:319-24. PMID: 15795927

22. Taylor DA, Fleming WW. Unifying perspectives of the mechanisms underlying the development of tolerance and physical dependence to opioids. J Pharmacol Exp Ther 2001;297:11-8. PMID: 11259522

23. Costa-Mattioli M, Sossin WS, Klann E, Sonenberg N. Translational control of long-lasting synaptic plasticity and memory. Neuron 2009;61:10-26. PMID: 19146809

24. Asante CO, Wallace VC, Dickenson AH. Mammalian target of rapamycin signaling in the spinal cord is required for neuronal plasticity 
and behavioral hypersensitivity associated with neuropathy in the rat. J Pain 2010;11:1356-67.

PMID: 20452291

25. Geranton SM, Jimenez-Diaz L, Torsney C, Tochiki KK, Stuart SA, Leith JL, Lumb BM, Hunt SP. A rapamycin-sensitive signaling pathway is essential for the full expression of persistent pain states. J Neurosci 2009;29:15017-27. PMID: 19940197

26. Jimenez-Diaz L, Geranton SM, Passmore GM, Leith JL, Fisher AS, Berliocchi L, Sivasubramaniam AK, Sheasby A, Lumb BM, Hunt SP. Local translation in primary afferent fibers regulates nociception. PLoS One 2008;3:e1961. PMID: 18398477

27. Obara I, Tochiki KK, Geranton SM, Carr FB, Lumb BM, Liu Q, Hunt SP. Systemic inhibition of the mammalian target of rapamycin (mTOR) pathway reduces neuropathic pain in mice. Pain 2011;152:2582-95. PMID: 21917376

28. Feng T, Yin Q, Weng ZL, Zhang JC, Wang KF, Yuan SY, Cheng W. Rapamycin ameliorates neuropathic pain by activating autophagy and inhibiting interleukin-1beta in the rat spinal cord. J Huazhong Univ Sci Technolog Med Sci 2014;34:830-7. PMID: 25480578

29. Jiang F, Pang XY, Niu QS, Hua LM, Cheng M, Ji $\mathrm{YH}$. Activation of mammalian target of rapamycin mediates rat pain-related responses induced by BmK I, a sodium channel-specific modulator. Mol Pain 2013;9:50. PMID: 24099268

30. Ferrini F, Trang T, Mattioli TA, Laffray S, Del'Guidice T, Lorenzo LE, Castonguay A, Doyon N, Zhang W, Godin AG, Mohr D, Beggs S, Vandal K, Beaulieu JM, Cahill CM, Salter MW, De Koninck Y. Morphine hyperalgesia gated through microglia-mediated disruption of neuronal $\mathrm{Cl}(-)$ homeostasis. Nat Neurosci 2013;16:183-92. PMID: 23292683

31. Liang DY, Li X, Clark JD. 5-hydroxytryptamine type 3 receptor modulates opioid-induced hyperalgesia and tolerance in mice. Anesthesiology 2011;114:1180-9. PMID: 21368652

32. Little JW, Cuzzocrea S, Bryant L, Esposito E, Doyle T, Rausaria S, Neumann WL, Salvemini D. Spinal mitochondrial-derived peroxynitrite enhances neuroimmune activation during morphine hyperalgesia and antinociceptive tolerance. Pain 2013;154:978-86. PMID: 23590939 